\title{
Quantitative Gas Chromatography, Using Retention Times
}

\author{
A. E. BRANDT and W. E. M. LANDS, Department of Biological Chemistry, \\ The University of Michigan, Ann Arbor, Michigan 48104
}

\section{ABSTRACT}

Diffusion of an injected sample within a gas chromatographic column does not begin from a point source but from a band. Therefore the method of calculating relative areas by using retention time $x$ peak height may require a correction factor to give a more accurate estimate of peak areas. When this correction was applied, the analysis was comparable with that obtained by the more time-consuming triangulation method.

\section{INTRODUCTION}

$\mathrm{T}$ HE RELATIVE PEAK areas obtained in gas chromatography have often been quantitatively determined by triangulation. One modification of the simple one-half (base $\times$ peak height) calculation uses the width at halfheight $\times$ peak height to give values that are less influenced by the difficulty of overlapping at the base of the peaks. Another method of quantitatively determining the relative areas uses the product of retention time $x$ peak height as described by Carroll (1). This method was suggested by Pecksok (2) and used also by others $(3,4)$. In the authors' opinion, this method has advantages over both the triangulation method and the seldom used paper-weighing method.

We have routinely used retention times $x$ peak height in calculating the approximate sample compositions by gas chromatography. As we developed a system for optimum speed and efficiency in analyzing methyl esters of long-chain fatty acids, this method led to low, calculated recoveries of the shorter derivatives. The alternate method of calculation, by triangulation, gave peak areas proportional to the component mass and indicated that the detector response was not appreciably different for the different esters. Thus there was something inadequate in the method that was used for estimating compositions with the aid of retention times. In order to improve the estimated areas and component mass values by the simpler peak-height $\times$ retention-time method, we considered corrections which would allow this method to give results comparable with those obtained by triangulation.

\section{EXPERIMENTAL PROCEDURE}

Two standard mixtures of methyl esters for gas-liquid chromatography (GLC) were obtained from The Hormel Institute, Austin, Minn., which had the following weight percentage compositions: Standard No. 3-8:0, $20.04 ; 10: 0,19.99 ; 12: 0,19.99 ; 14: 0,19.99$; 16:0, 19.99; Standard No. 5-16:0, 24.99; $18: 0,24.98 ; 20: 0,24.99 ; 22: 0,25.04$. Standard No. $3(100 \mathrm{mg})$ was dissolved in $10 \mathrm{ml}$ of carbon disulfide (reagent grade); Standard No. $5(100 \mathrm{mg})$ was dissolved in $10 \mathrm{ml}$ of hexane (reagent grade). All solvents gave only the solvent peak when checked by GLC for trace components.

The gas chromatograph was a Barber-Coleman Model 10, equipped with a hydrogen flame detector and operated at $280 \mathrm{C}$. The column was a 6 -ft $\times 1 / 4$-in glass column, packed with $10 \%$ diethyleneglycol succinate on Chromosorb P (Applied Science Laboratories) and maintained at a constant temperature of 195C. The flash heater was set at 225C. Argon carrier gas was used with a flow-meter value of $60 \mathrm{ml} / \mathrm{min}$ (based on a standard calibration of the meter with air). Samples (approx. $1 \mu 1)$ of each standard were injected onto the column with a $10-\mu 1$ syringe containing 1.5 $\mu l$ carbon disulfide as flushing solvent, separated from the plunger and sample by one$\mu l$ air spaces. At the time of injection the pen on the recorder was simultaneously moved so

TABLE I

Average Peak Widths at Half-Height and Retention Timesa

\begin{tabular}{ccc}
\hline Sample & Peak width & Retention time \\
\hline Standard No. 3 & sec & sec \\
$8: 0$ & $3.1 \pm 0.1$ & $37.1 \pm 0.2$ \\
$10: 0$ & $3.6 \pm 0.07$ & $47.4 \pm 0.2$ \\
$12: 0$ & $4.6 \pm 0.09$ & $64.6 \pm 0.5$ \\
$14: 0$ & $6.1 \pm 0.08$ & $97.5 \pm 0.3$ \\
$16: 0$ & $8.7 \pm 0.1$ & $153 \pm 0.4$ \\
Standard No. 5 & & \\
$16: 0$ & $9.5 \pm 0.07$ & $153 \pm 0.4$ \\
$18: 0$ & $13.8 \pm 0.2$ & $251 \pm 2$ \\
$20: 0$ & $21.1 \pm 0.1$ & $420 \pm 1$ \\
$22: 0$ & $33.8 \pm 0.3$ & $717 \pm 2$ \\
\hline
\end{tabular}

aThe results for No. 3 are averages of 10 injections and those for No. 5, of six injections (sample size, approximately $10 \mu \mathrm{g}$ ). In each case the measure of variance is the standard deviation. The chart speed of $2 \mathrm{in.} / \mathrm{min}$ gave recorded chromatograms in which $1 \mathrm{~mm}$ corresponded to $1.18 \mathrm{sec}$. 


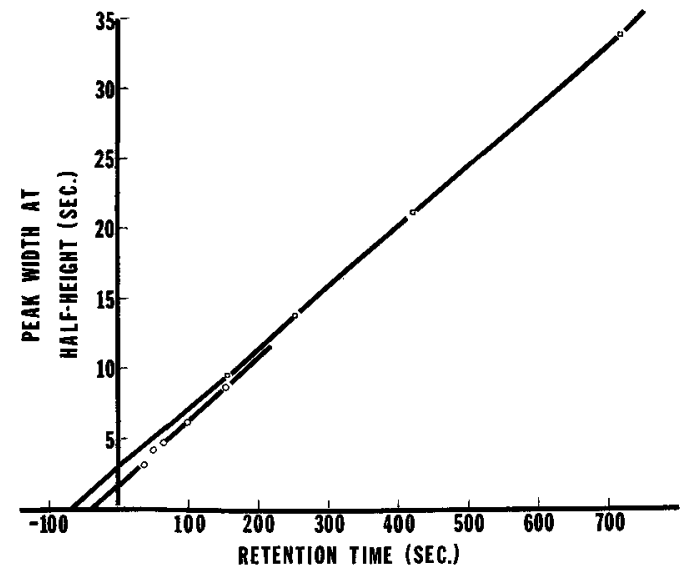

FIG. 1. Peak width at half-height on GLC of methyl esters of long-chain fatty acids vs. the retention time. The $\square$ is standard mixture No. 5; $\bigcirc$ is standard mixture No. 3 .

that the injection point was accurately known. Uncorrected retention times were measured from this point of injection.

\section{RESULTS}

The uncorrected retention times and peak widths at half-height which were determined are summarized in Table I. The height of the deflection from base line was also determined, but this varied depending upon the amount of standard injected and is not constant from injection to injection. The values for the width of the peaks at half-height for repeated injections of a given sample were easily reproducible and ranged from 3.1 to $33.8 \mathrm{sec}$ with the methyl esters used.

The relationship between peak width at halfheight and the retention time for that peak is shown in Fig. 1. The points lie on a straight line that does not go through the origin. In all cases that we have examined, the extrapolated value for the width at half-height at zero time has a finite positive value, ranging from 0.6 to $3.5 \mathrm{sec}$. Alternatively the line defines an imaginary or virtual injection point which would lie 27 to $72 \mathrm{sec}$ before the real injection point.

Sample calculations of relative peak areas by three methods are shown in Table II. The first method (A) represents the widely used procedure of triangulation and has consistently provided good results with reference standards. The second method (B) uses the product of the retention time and peak amplitude, both of which are conveniently and accurately measured. The third method $(C)$ uses the product of virtual retention time and peak amplitude on the basis that the virtual retention time is directly proportional to the peak width at halfheight.

The results from these calculations are shown in Table III. A measure of the error of measurement, indicated by the standard devia-

TABLE III

Comparison of Three Methods of Calculationa

\begin{tabular}{cccc}
\hline Sample & (A) & (B) & (C) \\
\hline Standard No. 3 & $\%$ & $\%$ & $\%$ \\
8:0 & $17.7 \pm 1.0$ & $13.6 \pm 0.2$ & $17.3 \pm 0.3$ \\
$10: 0$ & $19.5 \pm 0.4$ & $17.5 \pm 0.1$ & $19.6 \pm 0.1$ \\
$12: 0$ & $20.3 \pm 0.4$ & $20.1 \pm 0.2$ & $20.7 \pm 0.1$ \\
$14: 0$ & $21.3 \pm 0.2$ & $23.1 \pm 0.3$ & $20.9 \pm 0.2$ \\
$16: 0$ & $21.4 \pm 0.3$ & $25.8 \pm 0.3$ & $21.5 \pm 0.3$ \\
& & & \\
Standard No. 5 & & & \\
$16: 0$ & $25.3 \pm 0.2$ & $21.7 \pm 0.2$ & $25.3 \pm 0.3$ \\
$18: 0$ & $25.5 \pm 0.3$ & $24.5 \pm 0.2$ & $25.2 \pm 0.2$ \\
$20: 0$ & $25.2 \pm 0.8$ & $26.6 \pm 0.2$ & $25.1 \pm 0.1$ \\
$22: 0$ & $24.2 \pm 0.2$ & $27.3 \pm 0.1$ & $24.3 \pm 0.2$
\end{tabular}

aThe three methods $A, B$, and $C$ correspond to those described in Table II. The values are averages of six to 10 analyses.

TABLE II

Calculations of Relative Peak Areas

\begin{tabular}{|c|c|c|c|c|c|c|c|c|c|c|c|}
\hline Sample & $\begin{array}{l}\text { Peak } \\
\text { height } \\
\text { (1) }\end{array}$ & $\underset{(2)}{\operatorname{Amp} .^{a}}$ & $\begin{array}{c}\text { Peak } \\
\text { width } \\
(3)\end{array}$ & $\begin{array}{l}\text { Retentio } \\
\text { observed } \\
\text { (4) }\end{array}$ & $\begin{array}{l}\text { n time } \\
\text { virtual } \\
(5)\end{array}$ & $\begin{array}{c}\text { (A) } \\
(1) \times(2) \times(3)\end{array}$ & $\begin{array}{l}\text { Per } \\
\text { cent }\end{array}$ & $\begin{array}{l}\text { Calculated } \\
\text { (B) } \\
\text { (1) } \times(2) \times(4)\end{array}$ & $\begin{array}{l}\text { areas } \\
\text { Per } \\
\text { cent }\end{array}$ & $\frac{\text { (C) }}{(1) \times(2) \times(5)}$ & $\begin{array}{l}\text { Per } \\
\text { cent }\end{array}$ \\
\hline & $\mathrm{cm}$ & & $\mathrm{mm}$ & $\mathrm{cm}$ & $\mathrm{cm}$ & $\mathrm{cm}^{2}$ & & $\mathrm{~cm}^{2}$ & & $\mathrm{~cm}^{2}$ & \\
\hline $8: 0$ & 17.2 & 1.0 & 2.8 & 3.11 & 5.41 & 48.1 & 17.2 & 53.4 & 13.8 & 93.0 & 17.2 \\
\hline $10: 0$ & 16.8 & 1.0 & 3.2 & 3.97 & 6.27 & 53.7 & 19.2 & 66.7 & 17.2 & 105.3 & 19.4 \\
\hline $12: 0$ & 14.1 & 1.0 & 4.1 & 5.53 & 7.83 & 57.8 & 20.6 & 77.9 & 20.1 & 110.3 & 20.4 \\
\hline $14: 0$ & 10.8 & 1.0 & 5.5 & 8.28 & 10.58 & 59.5 & 21.2 & 89.7 & 23.1 & 114.6 & 21.2 \\
\hline $16: 0$ & 7.7 & 1.0 & 7.9 & 12.95 & 15.25 & 61.2 & 21.8 & 100.4 & 25.9 & 118.2 & 21.8 \\
\hline $16: 0$ & 9.69 & 1.0 & 7.9 & 12.9 & 18.1 & 71.4 & 25.3 & 116.4 & 21.6 & 163.9 & 25.1 \\
\hline 18:0 & 21.0 & 0.3 & 11.1 & 21.1 & 26.3 & 69.9 & 24.7 & 132.9 & 24.7 & 165.9 & 25.4 \\
\hline $20: 0$ & 13.5 & 0.3 & 17.8 & 35.3 & 40.6 & 72.1 & 25.5 & 143.1 & 26.6 & 164.3 & 25.2 \\
\hline $22: 0$ & 8.08 & 0.3 & 28.6 & 60.3 & 65.6 & 69.3 & 24.5 & 146.2 & 27.2 & 159.0 & 24.3 \\
\hline
\end{tabular}

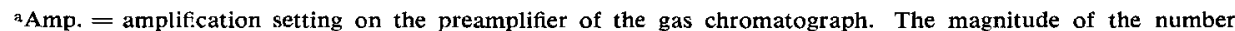
indicates the amount of detector signal needed for a full-scale response of the recorder. 
tions, was greatest when the peak width at half-height must be measured (A) since even the thickness of the pen tracing affects the small distances that must be measured. The leading edge of the pen tracing was consistently measured for uniform routine determinations. Yet the systematic error in composition with low values for short-chain acids and high values for the larger ones was most evident with method (B), in which only one acid in each series agreed with method (A). Although all three methods gave lower than theoretical values for octanoate, the other acids were adequately determined with methods (A) and (C).

The standards were diluted ( 1 to 10 ) in CS, and were injected to see if the peak widths at half-height and the virtual retention times could be decreased by this change in solvent and sample size. A small change was noted; the half-height width at zero time for Standard No. 3 changed from $1.3 \mathrm{sec}$ to $0.6 \mathrm{sec}$, and from $3.5 \mathrm{sec}$ to $2.4 \mathrm{sec}$ for Standard No. 5. These values correspond to shifts in the virtual injection point from $-25 \mathrm{sec}$ to $-12 \mathrm{sec}$ for Standard No. 3 and from -72 to $-52 \mathrm{sec}$ for Standard No. 5. The results in Table IV indicate that these changes lowered the required corrections but were not in themselves sufficient to produce similar values with methods (B) and (C).

\section{DISCUSSION}

The hydrogen flame-detector response gives uncorrected peak area percentage values that are approximately equivalent to the weight percentage $(5,6)$ with the series of long-chain fatty acids usually encountered. Additional factors have been proposed to compensate for differences in flame ionization-detector response (7) and for presumed destructive losses in the

TABLE IV

Effect of Sample Dilution on Calculated Areasa

\begin{tabular}{cccc}
\hline Sample & (A) & (B) & (C) \\
\hline Standard No. 3 & $\%$ & $\%$ & $\%$ \\
8:0 & $19.2(18.8)$ & $17.6(17.1)$ & $19.5(18.1)$ \\
$10: 0$ & $19.7(19.2)$ & $18.3(17.8)$ & $19.3(19.1)$ \\
$12: 0$ & $20.3(21.3)$ & $20.0(19.7)$ & $20.0(20.1)$ \\
$14: 0$ & $20.4(20.5)$ & $21.1(21.8)$ & $20.1(20.7)$ \\
$16: 0$ & $20.5(20.2)$ & $23.0(23.6)$ & $21.1(21.9)$ \\
& & & \\
Standard No. 5 & & & \\
$16: 0$ & $25.0(24.8)$ & $22.1(22.7)$ & $25.3(25.6)$ \\
$18: 0$ & $25.6(25.6)$ & $25.0(24.8)$ & $25.6(25.3)$ \\
$20: 0$ & $25.3(25.4)$ & $26.4(26.2)$ & $25.0(25.0)$ \\
$22: 0$ & $24.1(24.2)$ & $26.6(26.4)$ & $24.1(24.2)$ \\
& & &
\end{tabular}

aThe three methods $\mathbf{A}, \mathbf{B}$, and $\mathbf{C}$ are indicated in Table II. The results of duplicate analyses are in parentheses. column (8). Any dimension of the chromatogram that is directly proportional to peak width can give area percentage values that are equal to those from the triangulation method which uses a direct measurement of width. The characteristic property of gas chromatographic peaks of greater width in proportion to the amount of time the sample has been diffusing within the column makes the retention time helpful in calculating relative areas of symmetrical peaks. The results in Fig. 1 however indicate that the application of the sample to the column is not instantaneous but gives a band of material that may be distributed over a range of time from one to three $\mathrm{sec}$ (the equivalent peak width at zero retention time). Thus the diffusion within the column does not begin from a point source but rather from a band.

The width of the sample band in gas chromatography can be expected to depend upon the geometry of the injection chamber, rate of gas flow, flash-heater temperature (which would control the speed of solvent volatilization), and the volatility of the injected sample. Syringe techniques and speed of injection may also contribute one or two tenths of a second to the band width. These variables are all reasonably constant, finite properties of a given gas chromatographic system (including the operator); although their effects can be minimized, they cannot be eliminated.

We believe that one of the principal factors causing a wide initial band in our chromatograph is the rather large volume of the injection chamber of the commercially available columns. This space broadens the bands in much the same way that the "post-column dead volume" does (9). Our studies led to the realization that such factors as solute bandbroadening and plate-height abnormalities which are of concern to those who determine the height of the effective theoretical plate (HETP) in columns were indirectly involved in our quantitative estimations of percentage composition by the retention-time $\times$ peakheight method. The influence of injection time on the efficiency (or HETP) of GLC columns was indicated by Guiochon $(10,11)$, who noted that this factor "seems to have been completely neglected." He further indicated the magnitude of the contribution of injection time to zone spreading. The problem of initial sample band width was recently commented on by Bartlet and Mason (12), who found that the peak-height $\times$ retention-time method gave satisfactory results without corrections.

Our experience suggests that a major dif-

LiPIDS, Vol. 3, No. 2 
ficulty in satisfactorily applying the convenient retention-time $x$ height method may be due primarily to the injection system used when short retention times are involved. However the approximate band width at zero time can be estimated for a given chromatographic system and used to develop a simple correction factor for quantitative work even when the desire for faster analyses requires short retention times. Samples that are similar in size and composition to the unknowns which are analyzed should be employed in determining this correction factor. An understanding of this correction factor may be useful since it provides results as accurate as those obtained by triangulation and uses measurements that are more conveniently obtained.

\section{ACKNOWLEDGMENT}

This work was supported in part by grant AM-05310 from the U.S. Public Health Service.

\section{REFERENCES}

1. Carroll, K. K., Nature 191, 377-378 (1961).

2. Pecksok, R. L., "Principles and Practice of Gas Chromatography," John Wiley and Sons, Inc., New York, 1959, p 147.

3. Bartlet, J. C., and D. M. Smith, Can. J. Chem. 38, 2057-2065 (1960).

4. Tandy, R. K., F. T. Lindgren, W. H. Martin and R. D. Wills, Anal. Chem. 33, 665-669 (1961).

5. Ettre, L. S., and F. J. Kabot, J. Chromatog. 11, 114-116 (1963).

6. Moore, J. L., T. Richardson and C. H. Amundson, J. Gas Chromatog. 2, 318-319 (1964).

7. Ackman, R. G., and J. C. Sipos, JAOCS 41, 377378 (1964).

8. Ackman, R. G., J. C. Sipos and P. M. Jangaard, Lipids 2, 251-257 (1967).

9. Vandenheuvel, F. A., Anal. Chem. 35, 1193-1198 (1963).

10. Guiochon, G., Anal. Chem. 35, 399-400 (1963).

11. Gujochon, G., J. Gas Chromatog. 2, 139-145 (1964).

12. Bartlet, J. C., and J. L. Iverson, J. Assoc. Offic. Anal. Chem. 49, 21-27 (1966).

[Received June 13, 1967] 